\title{
Review of donor and recipient surgical procedures in lung transplantation
}

\author{
Duy C. Nguyen, Gabriel Loor, Philip Carrott, Alexis Shafii \\ Michael E. DeBakey Department of Surgery, Baylor College of Medicine, Houston, TX, USA \\ Contributions: (I) Conception and design: A Shafii; (II) Administrative support: G Loor; (III) Provision of study materials or patients: All authors; \\ (IV) Collection and assembly of data: All authors; (V) Data analysis and interpretation: All authors; (VI) Manuscript writing: All authors; (VII) Final \\ approval of manuscript: All authors. \\ Correspondence to: Duy C. Nguyen, MD. Baylor College of Medicine, One Baylor Plaza, BCM390, Houston, TX 77030, USA. \\ Email: dcnguyen@bcm.edu.
}

\begin{abstract}
Lung transplant remains the only durable treatment of end stage lung disease. Efficient surgical technique and preservation strategy are of critical importance in avoiding ischemia reperfusion injury and primary graft dysfunction (PGD), both associated with poorer outcomes. We present our surgical and preservation strategy for both donor and recipient procedures, including endobronchial and intracorporeal lung assessment, mediastinal dissection, lung preservation, donor pneumonectomy, back bench preparation, recipient approach, pneumonectomy, along with the bronchial, pulmonary artery, and pulmonary venous anastomoses.
\end{abstract}

Keywords: Lung; transplant; procurement; preservation

Submitted Apr 24, 2019. Accepted for publication Jun 11, 2019.

doi: $10.21037 /$ jtd.2019.06.31

View this article at: http://dx.doi.org/10.21037/jtd.2019.06.31

\section{Introduction}

This article provides a succinct overview of the surgical aspects of lung procurement and implantation. Recipient candidate selection, timing of listing, criteria for lung donor acceptability, size matching, donation after circulatory death, and ex vivo lung perfusion are discussed elsewhere.

The critical importance of surgical technique and preservation strategy lies in the association of prolonged ischemic times and poor donor lung preservation with ischemia reperfusion injury or primary graft dysfunction (PGD) $(1,2)$. PGD, in turn, is associated with increased mortality and chronic rejection. Severe PGD occurs in up to $30 \%$ of lung transplants within 72 hours (3). While various factors influence the development of PGD, optimizing surgical technique has an important role in minimizing its incidence and consequences.

Recipient age, body habitus, presence of pulmonary hypertension, and frailty amongst other characteristics each influence short and long-term outcomes as well. Familiarity with the various techniques and surgical approaches for lung transplantation allows tailoring of the operation for individual patient features. Herein, we detail the methods for donor lung procurement and discuss the techniques for recipient implantation.

\section{Donor procedures}

\section{Donor assessment checklist}

Donor procurement is often performed by junior staff in stressful, unfamiliar environments. Therefore, we feel that it is essential to have a formalized donor assessment checklist to detect and prevent adverse events. At our institution, we use the Donor Assessment Checklist developed between the Cleveland Clinic and the University of Minnesota (4).

\section{Initial donor evaluation}

\section{Endobronchial lung assessment}

Prior to procurement, flexible bronchoscopy is performed to assess endobronchial anatomy, secretions, or foreign 
material that has been aspirated. Findings of anatomical abnormalities such as a tracheal bronchus or pig bronchus (bronchus suis) would preclude usage of the lung. Purulent secretions commonly found in donor airways usually do not represent a serious infection and are typically cleared by bronchoscopic suctioning. The reappearance, or pooling, of purulent secretions after bronchoscopic evacuation is more concerning and requires evaluation of the lung parenchyma. Correlation of endobronchial findings with radiographs and computed tomography scans is helpful in establishing the severity of pneumonia in the donor lung. Bronchoalveolar lavage can be performed for microbiology, however positive cultures do not necessarily correlate with postoperative infections following lung transplant (5). Endobronchial findings should be relayed to the to the implanting team.

\section{Intracorporeal lung assessment}

A median sternotomy is performed in standard fashion and the pericardial sac is opened. Both pleural cavities are opened widely. Upon entering the pleural space, instantaneous deflation of the lung is a good indication of normal lung compliance (6).

The lungs are then assessed macroscopically by visualization and palpation to rule out masses, evaluate compliance, edema, emphysematous changes, contusions, and consolidations. The lungs should be palpated when the lungs are deflated to assess for nodules or masses. In donor patients, basilar and posterior atelectasis is commonly found and the intracorporeal assessment is an opportunity for recruitment maneuvers to clear atelectasis and aerate the lungs. Recruitment maneuvers include inflating the lungs with a Valsalva (airway pressure of $20 \mathrm{cmH}_{2} \mathrm{O}$ ) and then gently manipulating the lung to aerate the atelectatic regions. These maneuvers can increase $\mathrm{PaO}_{2} / \mathrm{FiO}_{2}$ ratios. It is important to note that a prolonged Valsalva with the concurrent manipulation of the lungs can cause hemodynamic instability. Some advocate avoiding the practice of sequentially eviscerating the lungs from the pleural spaces to minimize such hemodynamic instability, but rather perform recruitment in situ (7).

If there is any concern about the function of one lung or a particular lobe, selective pulmonary venous $\mathrm{PaO}_{2}$ measurements can be obtained from each of the four main pulmonary veins. Pulmonary vein blood gases are helpful in determining the acceptability of a unilateral lung when the systemic $\mathrm{PaO}_{2}$ may be suboptimal and an infectious process or injury has affected the contralateral lung. Four separate samples are taken from each pulmonary vein, appropriately labeled, and sent for blood gas analysis.

\section{Donor lung procurement}

\section{Mediastinal dissection}

The anatomical structures for both heart and lung procurement are isolated prior to systemic heparinization. However, overzealous manipulation of the heart can incite dysrhythmias. Routine intrapericardial dissection includes: (I) mobilization of the superior vena cava from the right pulmonary artery; (II) separation of the aorta from the main and right pulmonary arteries; and (III) opening of the oblique sinus separating the inferior vena cava from the right inferior pulmonary vein.

\section{Preservation solution}

While mediastinal dissection is being performed, ice cold preservation solution (Perfadex) is mixed and the infusion tubing is primed. Perfadex solution is an extracellular dextran-based, low potassium solution that reduces interstitial edema and maintains epithelial cell integrity. The topic of optimal preservation solution has been evaluated extensively. On a cellular level, high potassium preservation solutions resulted in decreased membrane potential hyperpolarization, more depolarization of the resting membrane potential, and decreased hyperpolarizationassociated relaxation of the pulmonary arterioles as compared to extracellular based low-potassium solution (Perfadex) (8). From a clinical standpoint, intracellular high-potassium solutions resulted in a higher incidence and severity of reperfusion injury as compared to the extracellular based low-potassium Perfadex solution (9). Additional studies have also identified higher alveolararterial oxygen ratio values and improved short and longterm post-transplant survival for donor lungs preserved with the extracellular based low-potassium Perfadex solution $(9,10)$.

\section{Lung preservation}

When all donor and recipient teams are ready, the patient is systemically heparinized. A purse-string suture is place in the pulmonary trunk. When the heart is also being procured, the site of cannulation should be closer to the bifurcation of the main pulmonary artery in order to maximize pulmonary trunk length for the heart recipient. A large bore cannula (20-22 French) is then inserted into the pulmonary trunk and secured with the purse-string suture. The cannula is de-aired and connected to the primed 
pulmoplegia tubing.

Prior to exsanguination and cross clamping of the aorta, 500 micrograms of prostaglandin are injected into the pulmonary trunk. Prostaglandin is a potent pulmonary vasodilator that counteracts the pulmonary vasoconstriction with cold pulmoplegia and improves perfusion throughout the lung(s) (11).

The donor is then exsanguinated by partially transecting the inferior vena cava and amputating the left atrial appendage. Alternatively, the left heart can be drained thru the left atrium at Sondergaard's groove. The superior vena cava is ligated using a silk ligature or vascular clamp. Shed blood is cleared by multiple field suctions. The aorta is then cross clamped with a large vascular clamp. Pulmoplegia flush is then initiated by gravity dependent flow. Simultaneously cardioplegia is administered thru the ascending aorta if the heart is also being procured.

It is important that the lungs continue to be ventilated with low tidal volumes and an $\mathrm{FiO}_{2}$ of $100 \%$. The pleural spaces are packed with ice slush. A total of 4-5 liters of Perfadex is administered antegrade, sufficient flush is evident when the left atrial effluent becomes clear colored.

\section{Donor pneumonectomy}

Once all preservation solution has been completed, the heart is excised in standard fashion with special attention to preserving sufficient left atrial tissue. When the heart is not being procured for use, the lung procurement surgeon will perform cardiectomy. In stepwise fashion, the superior vena cava is divided with care to avoid the right main pulmonary artery lying posteriorly. Then the inferior vena cava transection is completed. Next, left atrium is incised in Sondergaard's groove and the incision is extended circumferentially thereby leaving an atrial cuff of tissue to accompany the lungs. Lastly, the aorta and main pulmonary artery are transected, the main pulmonary artery being transected just proximal to its bifurcation. Following cardiectomy, excision of the lung bloc is performed beginning with release of the inferior pulmonary ligaments. Excess pericardium anterior to the phrenic nerve is excised. The posterior pericardium caudad to the pulmonary veins is then incised exposing the esophagus. The dissection proceeds cephalad very close to the esophagus on the right side with a combination of blunt and sharp dissection extending to the azygous vein which is also divided. This dissection is repeated on the left side with careful attention to the left mainstem bronchus as it crosses over the descending thoracic aorta.
The plane between the esophagus and lower trachea is developed from caudad to cephalad. The trachea is then exposed anteriorly by transecting the brachiocephalic artery. Circumferential isolation of the mid trachea is accomplished by both scissors and blunt finger dissection. Care is given during this maneuver to avoid the esophagus lying directly posterior.

The endotracheal tube is then withdrawn to the upper trachea and a moderate-sized breath hold is performed to inflate the lungs. The mid trachea is then double stapled with a right-angled surgical stapler (TA-30) and sharply divided. Remaining attachments are released, and the lung bloc is extirpated and taken to the back bench for retrograde flush with additional preservation solution and packaging for transport.

\section{Back bench}

The lung bloc is placed into a large basin containing ice. Additional Perfadex solution is administered in a retrograde fashion through each of the pulmonary veins $(\sim 500 \mathrm{~mL}$ per pulmonary vein). Pulmonary blood clots (emboli) are frequently seen in the pulmonary artery effluent, manual palpation of the respective lobe encourages egress of these clots during retrograde flush of the pulmonary veins. Advantages of retrograde flush relate to the improved distribution of preservation solution to include flushing of the bronchial arteries. Improved surfactant function has also been implicated with retrograde administration of pulmoplegia (12).

At this point, if each lung is being allocated to different recipients, the lung bloc is split. The left atrial cuff and the main pulmonary artery are divided in the midline with scissors. The left main stem bronchus is then double stapled with a right-angle stapler (TA-30) and divided sharply.

\section{Organ packaging for transport}

The donor lung(s) are then placed in a sterile plastic bag containing cold Perfadex solution and sealed. Two additional layers of packaging with sterile plastic bags are required, sterile ice slush is added the outer bag. The organ is then placed into an ice cooler for transport and appropriately labeled. The donor lung(s) are then transported with the appropriate paperwork including $\mathrm{ABO}$ verification forms, recipient match identification documents, donor blood samples and donor lymph node samples. An alternative method for donor lung transportation involves the use of portable normothermic ex vivo lung perfusion which is discussed in a separate chapter. 


\section{Recipient procedures}

\section{Surgical approach}

Multiple approaches for lung transplant have been utilized, each with advantages and disadvantages. Familiarity with each approach permits the best access for each individual based on disease process, body habitus, and the need for cardiopulmonary bypass.

For bilateral lung transplant, the options include: bilateral anterolateral thoracotomies with transverse sternotomy (clamshell thoracotomy), sequential thoracotomies, and median sternotomy. Clamshell thoracotomy through the 4th intercostal space and division of the sternum provides full exposure to the heart and lungs, facilitates dissection, and allows easy conversion to cardiopulmonary bypass if needed. In cases where adhesions are anticipated including re-operative cases, this approach is preferred. Bilateral thoracotomy is an alternative that spares sternal transection, however this requires patient repositioning for the contralateral lung implant. Potential need for cardiopulmonary bypass would be complicated when this approach is used. Median sternotomy provides acceptable exposure for bilateral lung transplant; however, this approach is not feasible without the use of cardiopulmonary bypass. Furthermore, if adhesions are anticipated, access is limited to release posterior adhesions. Median sternotomy is preferred in patients where thoracotomy access is complicated such as obese patients and females with large pendulous breasts.

Single lung transplantation can be performed through a posterolateral (5th intercostal space) or anterolateral (4th intercostal space) thoracotomy. In most cases, single lung transplantation can generally be performed without cardiopulmonary bypass. A left-sided double lumen endotracheal tube is employed for lung isolation. Inhaled nitric oxide can be helpful during single lung ventilation in cases with moderate pulmonary hypertension. If the need for cardiopulmonary bypass were to arise, central aortic and right atrial cannulation is easily accomplished via right anterolateral thoracotomy. Peripheral femoral arterial and venous cannulation are utilized for a left thoracotomy approach requiring cardiopulmonary bypass.

\section{Pneumonectomy}

Once surgical exposure is established, pneumonectomy is undertaken. During bilateral lung transplantation, each lung is sequentially extirpated, and the donor lung implanted prior to proceeding with the contralateral side. Both intracardiac and extracardiac dissection of the hilar vessels facilitates the resection and provides additional vessel length for anastomosis. Once the vessels are isolated, they are ligated distally with a vascular stapler. The bronchus is then identified, surrounding lymphatic tissue and bronchial vessels are ligated with hemoclips or sutures. The airway is then sharply transected just proximal to the respective lobar bifurcation (secondary carina). Unnecessary dissection along the proximal bronchus should be avoided as to preserve its vascular supply. During the pneumonectomy, care must be given to avoiding injury to the phrenic nerves and vagus nerves. Additionally, the left recurrent laryngeal nerve can be damaged during dissection of the left main pulmonary artery proximal to the ligamentum arteriosum, and dissection is this region should be avoided.

\section{Donor lung preparation}

The donor lung bloc is unpackaged and placed in a sterile basin with cold saline slush. The left atrial cuff and the main pulmonary artery are split in the midline. The left main stem bronchus is divided proximally thereby separating the bloc into left and right lungs. The vascular structures are isolated and perivascular tissue is released from the pulmonary artery to provide additional length. Excess left atrial cuff tissue is excised. The bronchus is then cut sharply as short as possible (1-2 rings from the lobar bifurcation on the right, $2-3$ rings on the left). Tissue surrounding the bronchus should be preserved as to maintain the collateral blood flow into this peribronchial tissue for anastomotic healing. The lung is then placed into the recipient chest, appropriately oriented, and covered with sterile ice.

\section{Donor lung implantation}

\section{Bronchial anastomosis}

The bronchial anastomosis is performed first. Both interrupted and running suture methods are acceptable. Our preference is a running suture along the membranous portion of the airway (Figure 1) and multiple interrupted sutures anteriorly on the cartilaginous portion (Figure 2). We use 4-0 Prolene suture for this anastomosis, although PDS suture is commonly used by others. Post-transplant airway complications are not uncommon, with a reported incidence of over $10 \%$ (14). For this reason, we commonly buttress the anterior portion of the anastomosis with a piece of donor hilar adipose tissue. 


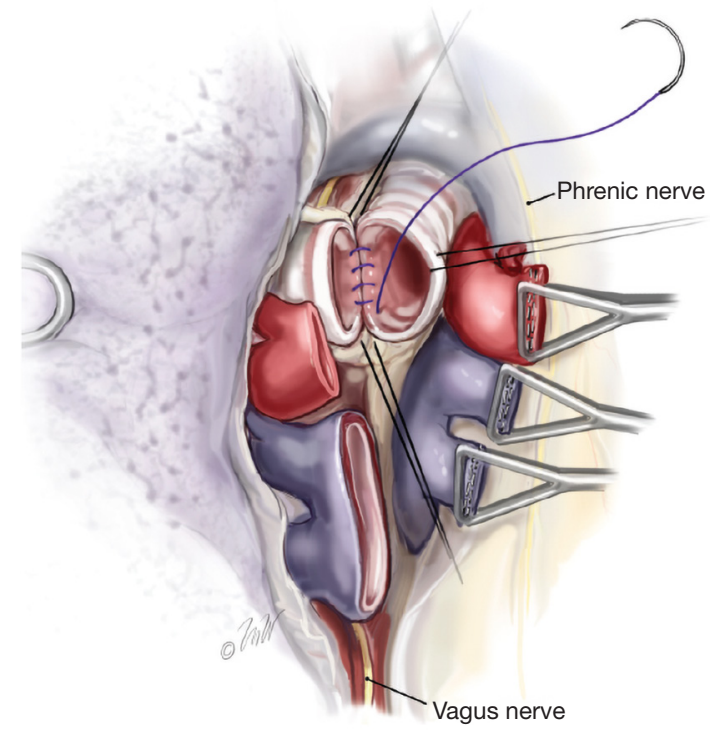

Figure 1 Posterior bronchial anastomosis (13).

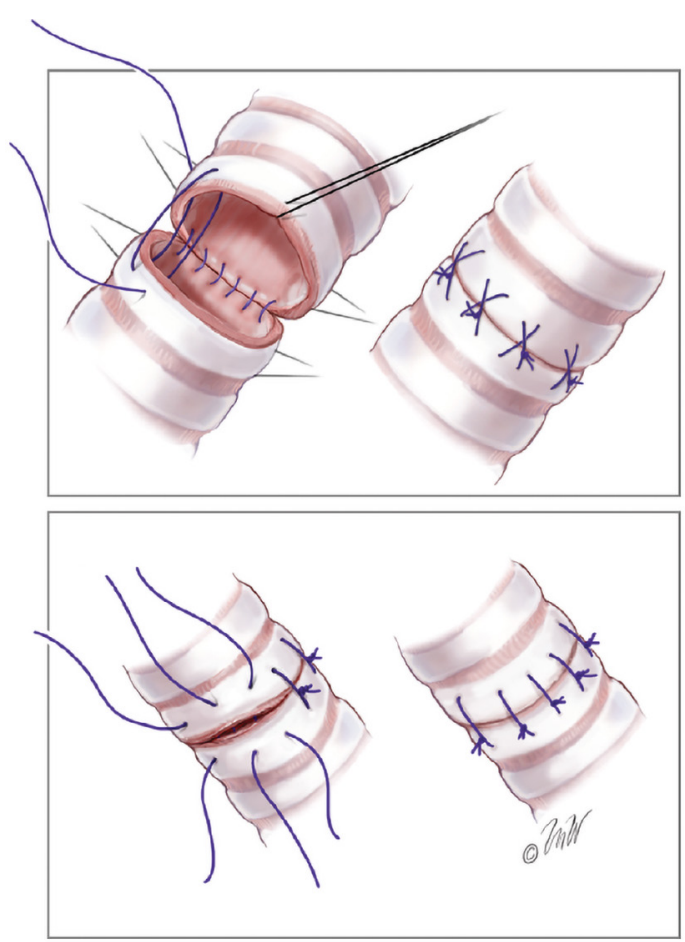

Figure 2 Anterior bronchial anastomosis (13).

\section{Pulmonary artery anastomosis}

The pulmonary artery is dissected as proximally as possible and a vascular clamp is applied carefully to avoid damage to the vessel. Over-vigorous closure of the vascular clamp

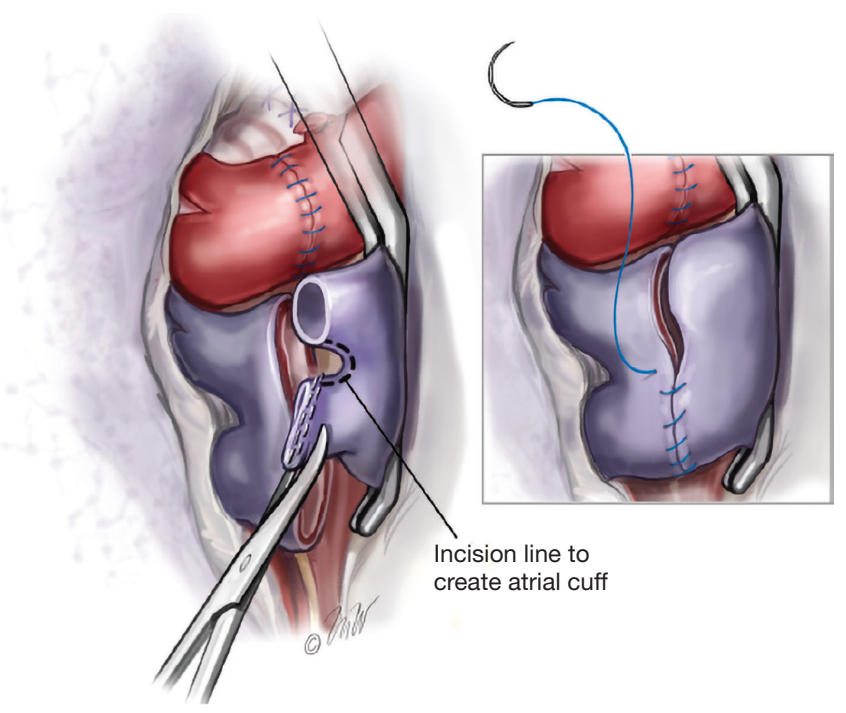

Figure 3 Pulmonary venous anastomosis (13).

can injure the fragile pulmonary artery. The staple line is then resected. In most cases, excess donor length of the pulmonary artery requires excision. Once the donor and recipient vessels are appropriately oriented, the pulmonary artery anastomosis is then performed with a running 5-0 Prolene suture.

\section{Pulmonary venous anastomosis}

The left atrium proximal to the pulmonary veins is controlled with a large angle vascular clamp (Satinsky clamp). The stapled portion of the pulmonary veins are amputated creating two openings in each pulmonary vein. The two openings are then connected by dividing the raphae of tissue thereby creating a recipient left atrial cuff (Figure 3). A left atrial cuff anastomosis is then performed with a running 4-0 Prolene suture. Commonly an imbricating technique is used for this anastomosis which allows an endocardial interface of the donor and recipient tissues. Once the anastomosis is completed, the suture is left untied for de-airing.

\section{Controlled reperfusion}

Prior to tying the completed left atrial anastomosis suture, the pulmonary artery clamp is released, and the implanted lung is de-aired. Once blood emanates from the suture line and air has cleared, the Satinsky clamp is released and the left atrial suture is tied. When the transplant is being performed without cardiopulmonary bypass, the 
pulmonary artery clamp is partially released as to slowly reperfuse the lung. Gradual reperfusion over 5-10 minutes to allow reperfusion of the lung in a controlled fashion can be helpful in reducing acute reperfusion injury (15). For transplants being performed with cardiopulmonary bypass, the pulmonary artery can be fully unclamped since blood flow thru the pulmonary vasculature is minimized while the heart is fully decompressed on cardiopulmonary bypass.

\section{Post-transplant ventilation}

Following completion of the lung implantation, the contralateral side is performed in a similar manner when a bilateral lung transplant is being performed. The lungs are then ventilated with low tidal volumes $(6-8 \mathrm{~mL} / \mathrm{kg})$ during which it is important to assure full expansion of all lobes by careful inspection. Additionally, proper orientation of the lobes should be verified to exclude lobar torsion in the inflated lung. A moderate level of PEEP $\left(8-10 \mathrm{cmH}_{2} \mathrm{O}\right)$ is instituted to assist with alveolar recruitment. Higher levels of PEEP may be required when hypoxia or interstitial pulmonary edema persists. Minimizing the $\mathrm{FiO}_{2}$ early after reperfusion is important as the newly implanted lung is susceptible to free radical injury (16). Inspired oxygen levels are reduced to a level required to maintain oxygen saturations $>95 \%$. Additional adjuncts to these ventilation strategies include the addition of inhaled nitric oxide as well as inotropic agents, both of which can effectively improve systemic oxygen saturations.

\section{Conclusions}

Lung transplant remains the only durable and efficacious treatment of end stage lung disease. Careful attention to detail in the evaluation, procurement, preservation and implantation of transplanted lungs can hopefully minimize subsequent complications in these complex patients. Standardization of procedures and gentle handling of the donor organ may help lower the rates of PGD. We have discussed methods we use in adjusting to the needs of individual patients, as well as expediting the surgery and reducing ischemic time. Careful assessment and reassessment of procedures surrounding the lung transplant operation can have a positive impact on outcomes.

\section{Acknowledgments}

None.

\section{Footnote}

Conflicts of Interest: The authors have no conflicts of interest to declare.

Ethical Statement: The authors are accountable for all aspects of the work in ensuring that questions related to the accuracy or integrity of any part of the work are appropriately investigated and resolved.

\section{References}

1. Kuntz CL, Hadjiliadis D, Ahya VN, et al. Risk factors for early primary graft dysfunction after lung transplantation: a registry study. Clin Transplant 2009;23:819-30.

2. de Perrot M, Bonser RS, Dark J, et al. Report of the ISHLT Working Group on Primary Lung Graft Dysfunction part III: donor-related risk factors and markers. J Heart Lung Transplant 2005;24:1460-7.

3. Diamond JM, Lee JC, Kawut SM, et al. Clinical risk factors for primary graft dysfunction after lung transplantation. Am J Respir Crit Care Med 2013;187:527-34.

4. Loor G, Shumway SJ, McCurry KR, et al. Process Improvement in Thoracic Donor Organ Procurement: Implementation of a Donor Assessment Checklist. Ann Thorac Surg 2016;102:1872-7.

5. Mattner F, Kola A, Fischer S, et al. Impact of bacterial and fungal donor organ contamination in lung, heart-lung, heart and liver transplantation. Infection 2008;36:207-12.

6. Puri V, Patterson GA. Adult lung transplantation: technical considerations. Semin Thorac Cardiovasc Surg 2008;20:152-64.

7. Pasque MK. Standardizing thoracic organ procurement for transplantation. J Thorac Cardiovasc Surg 2010;139:13-7.

8. Wu M, Yang Q, Yim AP, et al. Cellular electrophysiologic and mechanical evidence of superior vascular protection in pulmonary microcirculation by Perfadex compared with Celsior. J Thorac Cardiovasc Surg 2009;137:492-8.

9. Müller C, Fürst H, Reichenspurner H, et al. Lung procurement by low-potassium dextran and the effect on preservation injury. Munich Lung Transplant Group. Transplantation 1999;68:1139-43.

10. Ganesh JS, Rogers CA, Banner NR, et al. Steering Group of the UK Cardiothoracic Transplant Audit. Does the method of lung preservation influence outcome after transplantation? An analysis of 681 consecutive procedures. J Thorac Cardiovasc Surg 2007;134:1313-21. 
11. de Perrot M, Fischer S, Liu M, et al. Prostaglandin E1 protects lung transplants from ischemia-reperfusion injury: a shift from pro- to anti-inflammatory cytokines. Transplantation 2001;72:1505-12.

12. Strüber M, Hohlfeld JM, Kofidis T, et al. Surfactant function in lung transplantation after 24 hours of iscNhemia: advantage of retrograde flush perfusion for preservation. J Thorac Cardiovasc Surg 2002;123:98-103.

13. Sugarbaker DJ, Bueno R, Colson YL, et al. (eds). Adult Chest Surgery, 2nd. China Translation \& Printing, Ltd., 2009.

Cite this article as: Nguyen DC, Loor G, Carrott P, Shafii A. Review of donor and recipient surgical procedures in lung transplantation. J Thorac Dis 2019;11(Suppl 14):S1810-S1816. doi: $10.21037 /$ jtd.2019.06.31
14. Castleberry AW, Worni M, Kuchibhatla M, et al. A comparative analysis of bronchial stricture after lung transplantation in recipients with and without early acute rejection. Ann Thorac Surg 2013;96:1008-17; discussion 1017-8.

15. Pierre AF, DeCampos KN, Liu M, et al. Rapid reperfusion causes stress failure in ischemic rat lungs. J Thorac Cardiovasc Surg 1998;116:932-42.

16. Grace PA. Ischaemia-reperfusion injury. Br J Surg 1994;81:637-47. 